\title{
Use of a multi-vial mathematical model to design freeze-drying cycles for pharmaceuticals at known risk of failure
}

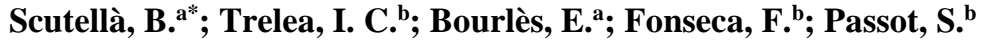

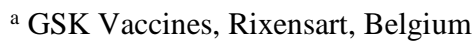 \\ b UMR GMPA, AgroParisTech, INRA, Université Paris Saclay, 78850 Thiverval-Grignon, France
}

*E-mail of the corresponding author: bernadette.z.scutella@gsk.com

\begin{abstract}
Freeze-drying is a dehydration method suitable for the stabilization of heatlabile pharmaceutical products, such as vaccines. Due to the vial-to-vial variability of heat and mass transfer during the process, the value of the critical process parameters (e.g., product temperature, sublimation rate) may be different between vials and batches often present significant product quality heterogeneity. The aim of this work was the development of a dynamic, multivial mathematical model making it possible to predict risk of failure of the process, defined as the percentage of vials potentially rejected by quality inspection. This tool could assist the design of freeze-drying cycle.
\end{abstract}

Keywords: lyophilization; vaccines; heat and mass transfer; mathematical modeling; design space 

failure

\section{Introduction}

Freeze-drying is a discontinuous process used to dry heat sensitive products by means of sublimation of the previously frozen product (primary drying), followed by desorption of the unfrozen water (secondary drying). Due to the use of low temperature, freeze-drying process is often the only solution to produce pharmaceutical and biological products (e.g., vaccines) with acceptable characteristics of stability, shelf-life, and potency. During the process design, two main constraints need to be taken into account in order to guarantee acceptable quality attributes of the final product, such as the visual aspect of the freeze-dried cake and the moisture content. Firstly, the product temperature has to be maintained below a critical value (namely collapse temperature) during the sublimation and desorption steps, to avoid the loss of the porous product structure. Furthermore, the desorption step should be carried out long enough to reach the target value of residual moisture content in the final product. However, due to the vial-to-vial variability of heat and mass transfer, the value of the critical process parameters (e.g., product temperature, sublimation rate and desorption rate) may be different between vials and the process can often result in vial batches presenting a significant heterogeneity in the product quality. Several mathematical models [1-5] were developed for the design of the primary drying and secondary drying steps. Most of these models were based on average values of the model parameters (e.g., product resistance, vial heat transfer coefficient, characteristic desorption time) over the vial batch and only few of them took into account the variability of the model parameters [2,3] due to differences in heat and mass mechanisms between vials processed in the same or different batches. The understanding and quantification of the mechanisms responsible for product quality variability can lead to a better prediction of the process parameters distribution and consequently of the risk of failure associated to the process.

Our goal in the present study was to develop a multi-vial dynamic mathematical model for freeze-drying for the process design, taking into account sources responsible for heat and mass transfer variability among vials. The developed model was then used to propose a new quality risk-based approach for the design of the primary and secondary drying steps of the freeze-drying process, which includes the evaluation of the percentage of vials potentially rejected for specific combinations of operating variables.

\section{Theory}

\subsection{Mathematical model}

The heat flux received during the process by the vial is assumed to mainly serve for the ice sublimation during primary drying and for the water desorption in secondary drying, the difference being responsible for temperature variation of product and vial in transient regimes. The evolution of product temperature $T_{p}$ (assumed to be the same as the vial) during primary and secondary drying can be determined as: 


$$
C_{p} \frac{d T_{p}}{d t}=\dot{Q}_{S V}-\Delta H_{s u b} \dot{m}_{s u b}-\Delta H_{d e s} \dot{m}_{d e s}
$$

\section{Heat transfer during primary drying}

The heat transfer between the shelf and the vial $\dot{Q}_{S V}$ depends on the temperature difference between the shelf $\left(T_{S}\right)$ and the product $\left(T_{p}\right)$, and can be expressed in term of vial heat transfer coefficient $K_{V}$ :

$$
\dot{Q}_{S V}=\left(\frac{1}{K_{V}}+\frac{l_{I}(t)}{\lambda_{i c e}}\right)^{-1} A_{B V}\left(T_{S}-T_{p}\right)
$$

The term $K_{V}$ depends on the vial bottom dimensions (vial-shelf contact area $A_{c}$ and depth of bottom curvature $l_{B V}$ ) and the chamber pressure $P_{C}$ and can be defined as:[6]

$$
K_{V}=C_{1} A_{c}+K_{r}+\frac{C_{2} P_{C}}{1+C_{2} \frac{l_{B V}}{\lambda_{v}} P_{C}}
$$

$K_{r}$ being the radiation contribution of the top and bottom shelf.[6]

\section{Mass transfer during primary drying}

During primary drying the sublimation flux can be defined by the difference between the equilibrium pressure at the ice-vapour interface $P_{v_{i}}$ and the partial vapour pressure in the chamber $P_{v_{C}}$ :

$$
\dot{m}_{s u b}=\frac{A_{i}}{R_{p}}\left(P_{v_{i}}-P_{v_{C}}\right)
$$

where $R_{p}$ is the the mass transfer resistance of the dried layer, which depends linearly on the dried layer thickness for the considered product:[7]

$$
R_{p}=R_{p_{0}}+l_{d} R_{p_{1}}
$$

$P_{v_{i}}$ can be calculated from the Clausius Clapeyron equation from the sublimation interface temperature,[6] and $P_{v_{C}}$ is defined as in Trelea et al.[8] assuming ideal gas law for water vapour in the drying chamber:

$$
\frac{d P_{v_{C}}}{d t}=\frac{R_{g} T_{C}}{V_{C} M_{W}}\left(\sum_{1}^{N_{V}}\left(\dot{m}_{s u b}+\dot{m}_{d e s}\right)-\dot{m}_{C N}\right)
$$

Finally, the fraction of ice $f$ contained in the product evolves with time as a function of the sublimation rate $\dot{m}_{\text {sub }}$ as:

$$
m_{0} \frac{d f}{d t}=-\dot{m}_{s u b}
$$


Use of a multi-vial mathematical model to design freeze-drying cycles for pharmaceuticals at known risk of failure

\section{Mass transfer during secondary drying}

The multilayer model developed by Trelea et al.[9] was used to describe the desorption kinetics in secondary drying.

\subsection{Heat and mass transfer variability in freeze-drying}

Four sources of inter-vial product quality variability were considered in this study:

> Vial geometry: $K_{V}$ is influenced by the shelf-vial contact area $A_{c}$ and the depth of bottom curvature $l_{B V}$ (Equation 3). Thus, differences in these dimensions among the vials can results in variability of the heat transfer among the vials on the shelf. In the present study, the distributions of $K_{V}$ at different chamber pressures over the vial batch were calculated based on the normal distributions of $A_{c}$ and $l_{B V}$, as previously proposed by Scutellà et al.[6]

$>$ Edge vial effect: It is well known that vials located at the periphery of the shelf receive an additional heat transfer by radiation from the wall and the rail and conduction through the gas between the chamber wall, the rail and the side wall of the vials. This phenomenon is known as edge vial effect. In the present study, the additional radiation heat flow rates from the wall and rail to the edge vials were calculated for edge vials using the Stefan-Boltzmann equation, as proposed in the 3D simplified radiation model developed by Scutellà et al. [10]

$>$ Mass transfer resistance: The product resistance variability in a large batch of vials was previously quantified as normal distributions of $R_{p_{0}}$ and $R_{p_{1}}$ in Scutellà et al.[7]

Desorption kinetics: The variability of the desorption kinetics was previously quantified as normal distributions of characteristics desorption times in Scutellà et al. [11]

\subsection{Prediction of the risk of vial rejection}

The developed mathematical model was used to propose a quality risk-based approach for the design of the primary and secondary drying steps of the freeze-drying process. The risk of failure of the primary drying step was calculated in terms of vials potentially rejected by considering two main constraints: (i) the product temperature had to be maintained at any time below a critical value (i.e., the glass transition temperature for amorphous products); (ii) the sublimation had to be completed at the end of primary drying (i.e., $f=0$ ). For the design of the secondary drying step, the constraints were: (i) the final moisture content had to be equal or lower than the target moisture content (1.5\%) and (ii) the temperature at any moment had to be lower than the glass transition temperature of the dry product. The range of acceptable combinations of operating variables (i.e., $T_{s}, P_{C}$, operating time) was then identified based on the target level of risk. The capability of the pilot freeze-dryer was considered to be not limiting regardless of the operating conditions.

\subsection{Numerical solution}

The developed model was solved using Matlab R2014b software provided of the Statistics Toolbox (The MathWorks, Inc., Natick, MA). The analysis of the effect of the heat and mass 
transfer variability on the critical process parameters during freeze-drying was performed using the Monte Carlo method. This method consisted in simulation of batches of 100 representative vials with random normal distributions of the considered model parameters $\left(A_{C}, l_{B V}, R_{p_{0}}, R_{p_{1}}, \tau_{r e f_{1}}\right.$ and $\tau_{r e f_{2}}$ ). Due to computer limitations, the number of simulated vials (100) was significantly smaller than the actual number of vials in the freeze-dryer (2310).

\section{Simulated system}

In the present work, the model was used to simulate freeze-drying process performed in the pilot freeze-dryer REVO (Millrock Technology, Kingston, United States). The equipment was composed of a drying chamber equipped with three shelves and a condenser running at temperature of $-75^{\circ} \mathrm{C}$. A total of 770 glass tubing vials (Müller + Müller, Holzminden, Germany) were supposed to be loaded on each shelf. The vials had a total volume of $3 \mathrm{~mL}$ and were all filled with $1.8 \mathrm{~mL}$ of $5 \%$ aqueous sucrose solution. Furthermore, the vial array was surrounded by a metallic rail (shielding $70 \%$ of the lateral wall of the vials). The shelf was considered to be at a temperature of $-50^{\circ} \mathrm{C}$ at the beginning of primary drying.

\section{Results}

The developed model was used to predict the risk of failure of the process in terms of percentage of vials potentially rejected. Potential vial rejection of the primary and secondary drying steps were predicted for different combinations of operating variables (shelf temperature, chamber pressure, operating time) to select the best cycle at the maximum allowed risk ( $1 \%)$. Figure $1 \mathrm{~A}$ presents the percentage of vial rejection due to a product temperature higher than $T_{g}^{\prime}$ observed performing a primary drying of 84 hours in function of the chamber pressure and shelf temperature. Typical ranges of chamber pressure and shelf temperature used in the pharmaceutical industry were explored, respectively between 4 and $10 \mathrm{~Pa}$, and $-40^{\circ} \mathrm{C}$ and $-10^{\circ} \mathrm{C}$. The results shows that, if a value of shelf temperature higher than $-27^{\circ} \mathrm{C}$ is applied during primary drying, the percentage of vial rejection become higher than $1 \%$ for most of the pressures tested. Furthermore, Figure 1B presents the percentage of vial rejection due to the sublimation not completed at the end of primary drying observed performing a primary drying step of 84 hours in function of the shelf temperature and chamber pressure. A shelf temperature higher than $-30{ }^{\circ} \mathrm{C}$ has to be considered to avoid the presence of ice at the end of the primary drying time. Thus, the use of a shelf temperature of $-27{ }^{\circ} \mathrm{C}$ and a chamber pressure between 4 and $9.3 \mathrm{~Pa}$ during primary drying step of $84 \mathrm{~h}$ results in a percentage of vial rejection lower than the maximum allowed one $(1 \%)$. Figure $2 \mathrm{~A}$ presents the risk of vial rejection associated to the secondary drying step due to a product temperature higher than $T_{g}$. A range of shelf temperatures between $0{ }^{\circ} \mathrm{C}$ and $40{ }^{\circ} \mathrm{C}$ was explored, and the pressure was maintained constant at $6 \mathrm{~Pa}$. The product temperature remains below the critical value regardless of the shelf temperature applied during the secondary 
drying step, because the product dries enough during the shelf temperature ramp $\left(1^{\circ} \mathrm{C} \mathrm{min}\right.$ ${ }^{1}$ ). Figure $2 \mathrm{~B}$ presents the risk of vial rejection of associated to the secondary drying step due to a final moisture content in the product higher than the target value of $1.5 \%$. A shelf temperature higher than $4{ }^{\circ} \mathrm{C}$ will lead to no vial rejection if secondary drying is carried for at least $45 \mathrm{~h}$. As product temperature remains below the critical value regardless the shelf temperature value used, the optimal operating conditions for secondary drying will be a shelf temperature of $40{ }^{\circ} \mathrm{C}$ and an operating time of $4 \mathrm{~h}$, which leads to a vials rejection lower than $1 \%$.
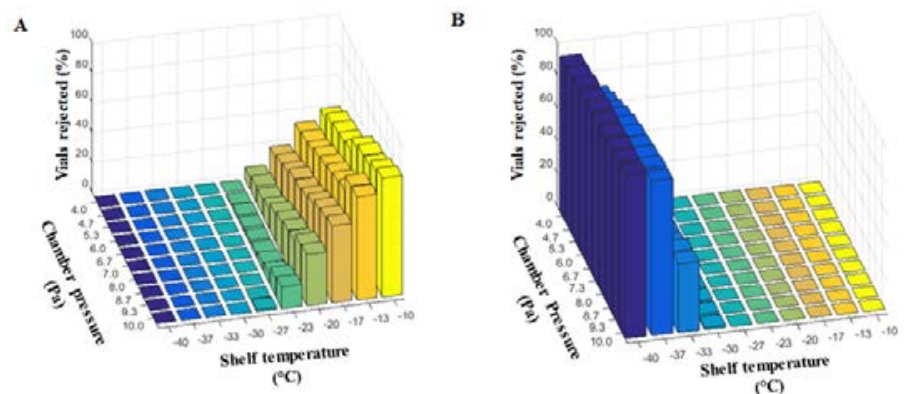

Fig. 1 Potential vial rejection (\%) in primary drying performed at different chamber pressures and shelf temperatures due to $(A)$ a product temperature higher than the critical value $\left(T_{g}^{\prime}\right)$ and (B) sublimation not completed after $84 \mathrm{~h}$ of primary drying.
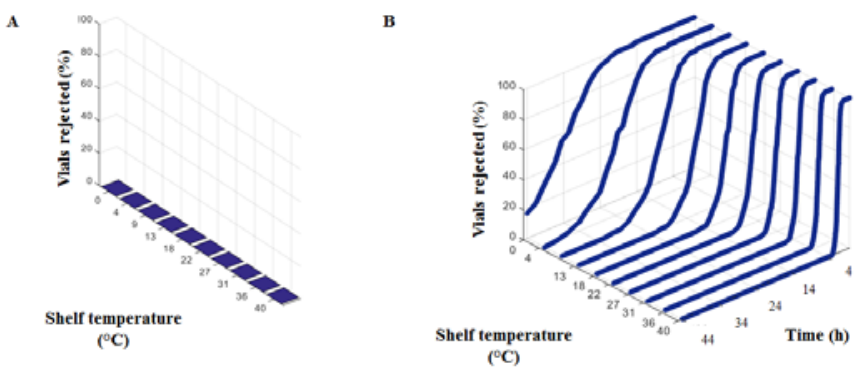

Fig. 2 Potential vial rejection (\%) in secondary drying due to (A) a product temperature higher than the critical value $\left(T_{g}\right)$ during the whole duration of the secondary drying step and (B) product moisture content higher than the target value (1.5\%) for different shelf temperatures and operating times.

\section{Conclusions and perspectives}

In this work, a multi-vial, dynamic mathematical model of the primary and secondary drying steps of the freeze-drying process was developed, including the heterogeneity of parameters such as the vial dimensions, the vial position on the shelf and the random nucleation process. The model was used to calculate the risk of failure of the primary and secondary drying steps for a $5 \%$ sucrose solution processed in a pilot freeze-dryer, expressed in terms of percentage of vials potentially rejected. In the future, the proposed model will be experimentally 
validated and used to calculate the design spaces of primary and secondary drying for the cycle transfer and scale-up of the process.

\section{Nomenclature}

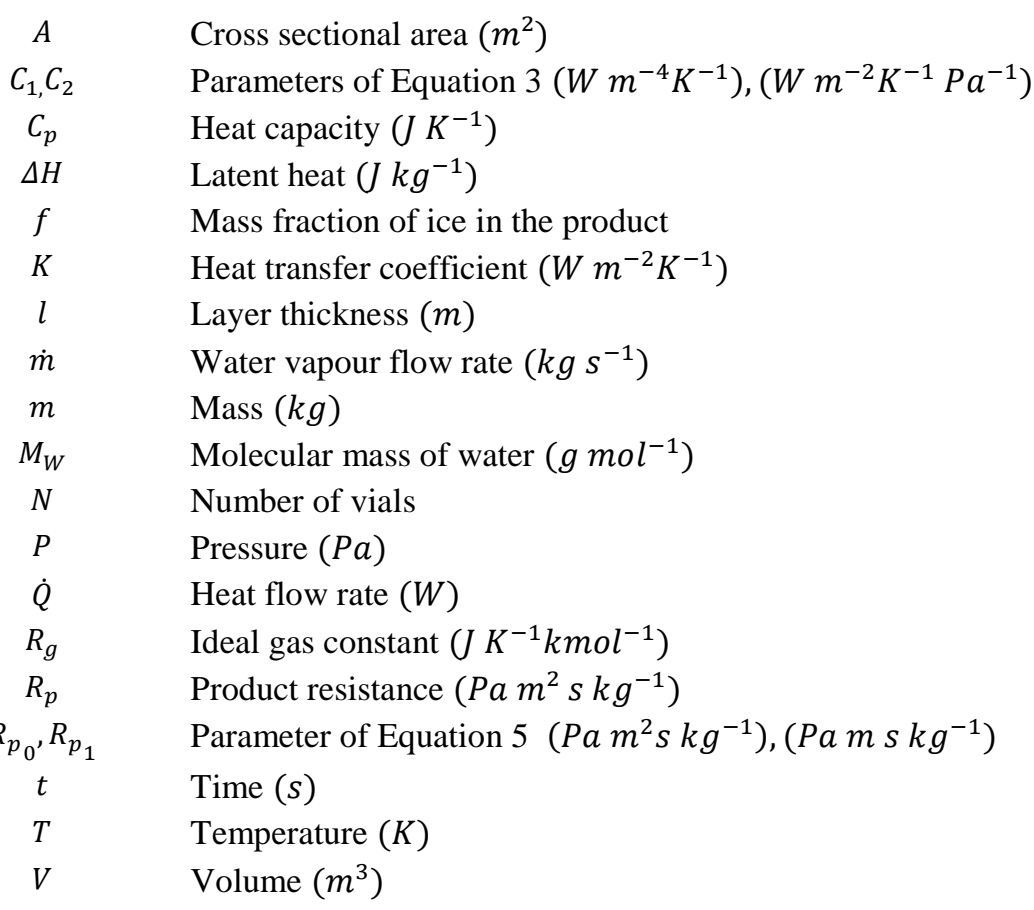

\section{Greek}

$\beta \quad$ Mass transfer parameter $\left(s \mathrm{~kg}^{-1} \mathrm{~K}^{-1}\right)$

$\lambda$ Thermal conductivity $\left(W \mathrm{~m}^{-1} \mathrm{~K}^{-1}\right)$

$\tau_{\text {ref }} \quad$ Characteristic desorption time at reference temperature $(s)$

\section{Subscripts and Superscript}

0

$B$

$C, C$

CN

$d$

des

$f$

$i$

ice

$P$

$r$

$S, s$
Initial

Bottom

Chamber and contact, respectively

Condenser

Dried

Desorption

Frozen

Interface

Ice

Product

Radiation

Shelf and solids, respectively 
Use of a multi-vial mathematical model to design freeze-drying cycles for pharmaceuticals at known risk of failure

$\begin{array}{cl}\text { sub } & \text { Sublimation } \\ T & \text { Top } \\ V, v & \text { Vial and vapour, respectively } \\ w & \text { Water }\end{array}$

Study Sponsorship: this work was sponsored by GlaxoSmithKline Biologicals SA which was involved in all stages of the study conduct and analysis.

Author Contributions: All authors contributed to developed the model, had full access to the data, were involved in drafting and critically revising the manuscript and finally approved the manuscript before submission.

Conflict of Interest: EB and BS are employees of the GSK group of companies. ICT's laboratory received a grant from GlaxoSmithKline Biologicals SA.

Acknowledgements: the authors would like to thank Yves Mayeresse and Benoit Moreau (GSK Vaccines) for reviewing this work.

\section{References}

[1] Pikal, M.J. Use of Laboratory Data in Freeze Drying Process Design: Heat and Mass Transfer Coefficients and the Computer Simulation of Freeze Drying. PDA Journal of Pharmaceutical Science and Technology 1985, 39, 115-139.

[2] Mortier, S.; Van Bockstal, P.J.; Corver, J.; Nopens, I.; Gernaey, K.V.; De Beer, T. Uncertainty analysis as essential step in the establishment of the dynamic Design Space of primary drying during freeze-drying. Eur J Pharm Biopharm 2016, 103, 71-83.

[3] Pisano, R.; Fissore, D.; Barresi, A.A.; Brayard, P.; Chouvenc, P.; Woinet, B. Quality by design: optimization of a freeze-drying cycle via design space in case of heterogeneous drying behavior and influence of the freezing protocol. Pharm Dev Technol 2013, 18(1), 280-295.

[4] Velardi, S.A.; Barresi A.A. Development of simplified models for the freeze-drying process and investigation of the optimal operating conditions. Chem Eng Res Des 2008, 86(1), 9-22.

[5] Gan, K.H.; Bruttini R.; Crosser O.K.; Liapis A.I. Freeze-drying of pharmaceuticals in vials on trays: effects of drying chamber wall temperature and tray side on lyophilization performance. Int J Heat Mass Transfer 2005, 48, 1675-1687.

[6] Scutella B.; Passot S.; Bourles E.; Fonseca F.; Trelea I.C. How Vial Geometry Variability Influences Heat Transfer and Product Temperature During Freeze-Drying. J Pharm Sci 2017, 106(3), 770-778.

[7] Scutellà B.; Trelea I.C.; Bourlés, E.; Fonseca, F.; Passot, S. Determination of the product resistance variability and its influence on the product temperature in pharmaceutical freezedrying. Eur J Pharm Biopharm. Submitted.

[8] Trelea, I.C.; Fonseca, F.; Passot, S.; Flick D. A Binary Gas Transport Model Improves the Prediction of Mass Transfer in Freeze Drying. Drying Technology 2015, 33(15-16), 18491858.

[9] Trelea, I.C.; Fonseca, F.; Passot, S. Dynamic modeling of the secondary drying stage of freeze drying reveals distinct desorption kinetics for bound water. Drying Technology 2015, 34(3), 335-345.

[10] Scutellà B.; Plana-Fattori A.; Passot S.; Bourlès E.; Fonseca F.; Flick D.; Trelea, I.C. 3D mathematical modelling to understand atypical heat transfer observed in vial freeze-drying. Appl Therm Eng 2017, 126, 226-236.

[11] Scutellà, B.; Bourlès, E.; Tordjman, C.; Fonseca, F.; Mayeresse, Y.; Trelea, I. C.; Passot, S. Can the desorption kinetics explain the residual moisture content heterogeneity observed in pharmaceuticals freeze-drying process? In proceedins of 6th European Drying Conference, Liegi, Belgium, July 19-21, 2017. 